\title{
0 agente comunitário de saúde e suas atribuições: os desafios para os processos de formação de recursos humanos em saúde
}

\author{
Community health agent and his attributions: challenges facing the training \\ process of human health resources
}

PALAVRAS-CHAVE: Assistência social; recursos humanos em saúde; Saúde da família.

KEY WORDS: Social assistance; health manpower; family health.

PALABRAS CLAVE: Asistencia social; recursos humanos en salud; salud de la familia.

${ }^{1}$ Enfermeira; Coordenadora Geral da Política de Recursos Humanos da Secretaria de Políticas do Ministério da Saúde.

Acredita-se que por serem (os agentes) pessoas do povo, não só se assemelham nas características e anseios deste povo, como também preenchem lacunas, justamente por conhecerem as necessidades desta população. Acredito que os agentes são a mola propulsora para a consolidação do Sistema único de Saúde, a organização das comunidades e a prática regionalizada e hierarquizada de assistência, na estruturação dos distritos sanitários. Ser agente de saúde é ser povo, é ser comunidade, é viver dia a dia a vida daquela comunidade.(...) É ser o elo de ligação entre as necessidades de saúde da população e o que pode ser feito para melhorar suas condições de vida. É ser a ponte entre a população e os profissionais e serviços de saúde. O agente comunitário é o mensageiro de saúde de sua comunidade. (Dirigente da Fundação Nacional de Saúde, Brasil, 1991, p.5)

$<$ joana.silva@saude.gov.br>

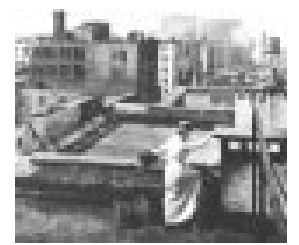

${ }_{2}^{2}$ Médica sanitarista, Centro de Saúde-Escola Samuel B. Pessoa - Universidade de São Paulo; coordenadora do Projeto São Remo. <csebp@usp.br> 
Ser agente comunitário de saúde é, antes de tudo, ser alguém que se identifica, em todos os sentidos, com a sua própria comunidade, principalmente na cultura, linguagem, costumes; precisa gostar do trabalho. Gostar, principalmente, de aprender e repassar as informações, entender que ninguém nasce com destino de morrer ainda criança ou de ser burro. Nós vivemos conforme o ambiente. É obrigação dos agentes comunitários de saúde lutar e aglomerar forças em sua comunidade, município, estado e país, em defesa dos serviços públicos de saúde, pensar na recuperação e democratização desses serviços, entendendo que é o serviço público que atende à população pobre; é preciso torná-lo de boa qualidade. Precisamos lutar por outros fatores que são determinantes para a saúde como: trabalho, salário justo, moradia, saneamento básico, terra para trabalhar e participação nas esferas de decisão dos serviços públicos. (Agente Comunitária de Saúde - Recife, Brasil, 1991, p.6)

\section{Introdução}

No Brasil, o Programa de Saúde da Família (PSF) pode ser visto como uma retomada de proposições contidas nas políticas públicas federais que estiveram em evidência, desde meados dos anos setenta, até início dos anos oitenta. Nesse sentido, destacam-se o Programa de Interiorização de Ações de Saúde e Saneamento (PIASS) - 1976 - e o Programa Nacional de Serviços Básicos de Saúde ( $7^{\text {a }}$ Conferência Nacional de Saúde, 1982), que visavam à extensão da cobertura e ampliação do acesso a serviços de saúde para grupos sociais ainda marginalizados (Donnangelo \& Pereira, 1976), moradores em regiões de baixa densidade populacional, ou pequenos centros urbanos da Região Nordeste, com condições de saúde muito precárias. Pode-se considerar, de certa forma, que essas políticas tiveram alguma continuidade em diversas iniciativas de âmbito regional ou local, $e$ que se traduziram em programas de tipo agentes comunitários de saúde, encampados pelo Ministério da Saúde como programa nacional, o PNACS, em 1991 (Jatene et al., 2000). Documentos do Ministério da Saúde, de 1997 e 1999, reforçam o entendimento do PSF como uma estratégia para organização da atenção básica à saúde no país (Brasil, 1997 e 1999a) e, a partir de 1998, para a realização dos princípios do SUS - a integralidade, a universalidade, a eqüidade.

Atualmente, esse tipo de Programa encontra-se em fase de expansão, tanto em áreas rurais como em centros urbanos. Além disso, esses modelos vêm sendo, cada vez mais, implantados em grandes cidades e áreas metropolitanas, mantendo, entre seus pressupostos e estratégias de intervenção básicos, as perspectivas de ampliação do acesso e de extensão de cobertura por serviços de saúde para parcelas específicas da população brasileira, de racionalidade técnica e econômica, de integralidade e humanização do atendimento, de participação popular em saúde, o estabelecimento de vínculos e a criação de laços de compromisso e de coresponsabilidade entre os profissionais de saúde e a população. 
Agente comunitário de saúde: a expectativa de atuação, as competências

Historicamente, a idéia que apoia a inserção do agente comunitário de saúde envolve um conceito que, sob as mais diferentes formas, nomenclaturas $e$ racionalidades, aparece em várias partes do mundo, ou seja, a idéia essencial de elo entre a comunidade e o sistema de saúde. Mais recentemente, no PSF, eles foram incluídos em equipes de trabalho que contam com um médico, um enfermeiro, um a dois auxiliares de enfermagem, com proposta de atuação para a unidade básica, o domicílio e a comunidade. Entretanto, se por um lado, a definição das atribuições tem sido um dos resultados mais efetivos das negociações locais, segundo as suas realidades, os seus contextos, os seus agentes, por outro lado, a partir de uma recuperação histórica analítica desse sujeito, de sua inserção, do seu trabalho - ações e interações - consegue-se apreender as principais idéias e conceitos subjacentes à proposição de agentes de saúde em cada um dos momentos e contextos históricos (Silva, 2001). Assim, desde a proposição do Auxiliar de Saúde, no PIASS, em 1976, até o Programa de Saúde da Família, com as especificidades de uma metrópole, em 2001, como agente comunitário de saúde, embora com graus variados da ênfase colocada, consegue-se identificar dois componentes ou dimensões principais da sua proposta de atuação: um mais estritamente técnico, relacionado ao atendimento aos indivíduos e famílias, a intervenção para prevenção de agravos ou para o monitoramento de grupos ou problemas específicos, e outro mais político, porém não apenas de solidariedade à população, da inserção da saúde no contexto geral de vida mas, também, no sentido de organização da comunidade, de transformação dessas condições. Este componente político expressa, na dependência da proposta considerada, duas expectativas diversas ou complementares: o agente como um elemento de reorientação da concepção e do modelo de atenção à saúde, de discussão com a comunidade dos problemas de saúde, de apoio ao auto-cuidado - dimensão mais ético-comunitária - e o agente como fomentador da organização da comunidade para a cidadania $e$ a inclusão, numa dimensão de transformação social. Um outro aspecto bastante encontrado na prática, mas não relacionado nas atribuições dos agentes de nenhuma das propostas, é a dimensão de assistência social. Assim, o agente aparece, nos diferentes

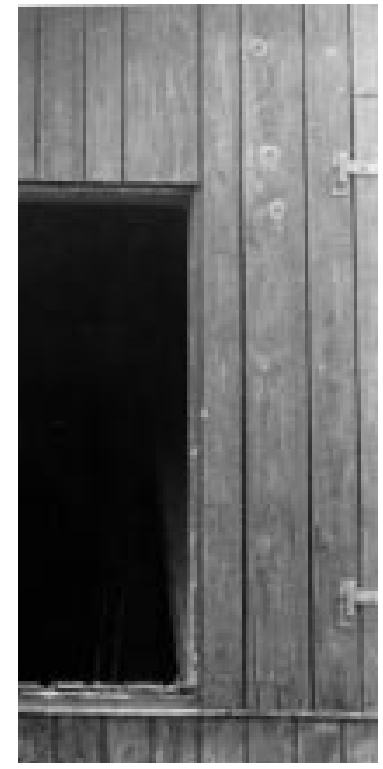
programas oficiais, como um personagem fruto de uma tentativa de juntar as perspectivas da atenção primária e da saúde comunitária, buscando resolver questões, como o acesso aos serviços, no que lhe corresponde de racionalidade técnica, mas também integrando as dimensões de exclusão $e$ cidadania, ou seja, o desafio de juntar o pólo técnico ao pólo político das propostas.

Nogueira e Ramos (2000) identificam, no trabalho do agente, a dimensão tecnológica $e$ a dimensão solidária e social, as quais consideram que têm, sempre, potenciais de conflitos. Essas dimensões expressam, possivelmente, os pólos político e técnico do Programa, acima referidos. Este é o dilema permanente do agente: a dimensão social convivendo com a dimensão técnica assistencial. Ao incorporar essas duas facetas em suas formulações, o conflito aparece principalmente na dinâmica da prática cotidiana. 
Determinados programas acabam dando mais espaço para um ou para o outro pólo; nenhum dos programas de saúde da família fez a síntese. No diaa-dia, os agentes são colocados diante de contradições sociais, o que é "muito pesado" e, por isso, eles fazem determinadas opções, segundo as exigências, as recompensas e suas referências.

Em estudo desenvolvido sobre o agente comunitário de saúde do Projeto QUALIS/PSF, no município de São Paulo, Silva (2001) identificou que o agente comunitário não dispõe de instrumentos, de tecnologia, aqui incluídos os saberes para as diferentes dimensões esperadas do seu trabalho. Essa insuficiência faz com que ele acabe trabalhando com o senso comum, com a Religião e, mais raramente, com os saberes e os recursos das famílias $e$ da comunidade. Há saberes de empréstimo para o pólo técnico, não os há para a dimensão considerada como mais política, nem há propostas ou trabalho consistente do "agir comunicativo".

Documento do Ministério da Saúde (Brasil, 1999b) enfatiza a necessidade de que, face o novo perfil de atuação para o agente comunitário de saúde, sejam adotadas formas mais abrangentes e organizadas de aprendizagem, $o$ que implica que os programas de capacitação desses trabalhadores devam adotar uma ação educativa crítica capaz de referenciar-se na realidade das práticas e nas transformações políticas, tecnológicas e científicas relacionadas à saúde e de assegurar o domínio de conhecimentos $e$ habilidades específicas para o desempenho de suas funções.

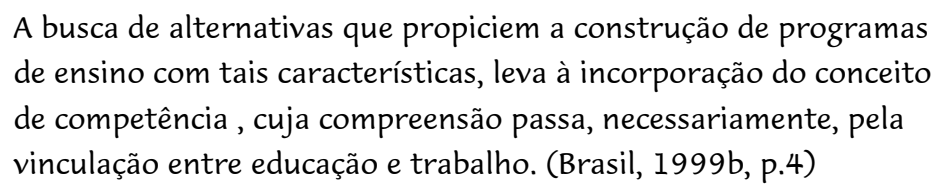

O conceito de competência é expresso como "a capacidade pessoal de articular conhecimentos, habilidades e atitudes inerentes a situações concretas de trabalho" (Brasil, 1999b, p.4).

Ainda o citado documento, propondo-se a subsidiar os Pólos de Capacitação do PSF, as Escolas Técnicas de Saúde do SUS e outras instituições participantes do esforço de preparação de recursos humanos para a estratégia de saúde da família, define, para o agente comunitário de saúde, sete competências: trabalho em equipe; visita domiciliar; planejamento das ações de saúde; promoção da saúde; prevenção e monitoramento de situações de risco e do meio ambiente; prevenção $e$ monitoramento de grupos específicos; prevenção e monitoramento das doenças prevalentes; acompanhamento e avaliação das ações de saúde. A complexidade e a dimensão dos desafios colocados podem ser ilustradas quando se toma a caracterização, por exemplo, da competência Promoção da Saúde:

capacidade para participar da promoção da saúde, na sua área de abrangência, através do desenvolvimento de trabalho educativo, do estímulo à participação comunitária e do trabalho intersetorial, com o objetivo da qualidade de vida. (Brasil, 1999b, p.16) 
Algumas questões logo aparecem: que saber é necessário para esse saber fazer? Como garantir esse saber que não é apenas o da saúde?

Outras dimensões importantes a considerar quando se discutem as expectativas de atuação do agente comunitário de saúde e os desafios para os processos de preparação desse "novo" perfil, referem-se aos mecanismos de seleção, aos processos de capacitação, aí incluídos os treinamentos introdutórios e a educação continuada $e$ a sistemática de supervisão adotada.

O saber científico que orienta a Medicina $e$ as áreas correlatas, ao ser aplicado a situações concretas desdobra-se em saber operante, uma vez que deve dar conta de outras necessidades não recobertas pela ciência, como os valores, as condições de vida, as relações afetivas (Mendes-Gonçalves, 1994). No trabalho do agente, no realizar ações e interações, há uma série de situações para as quais a área da saúde ainda não desenvolveu nem um saber sistematizado nem instrumentos adequados de trabalho e gerência, que compreendam desde a abordagem da família, o contato com situações de vida precária que determinam as condições de saúde, até o posicionamento frente à desigualdade social e a busca da cidadania. Para dar conta da elaboração do saber a partir do trabalho (Abbott, 1990), os espaços de supervisão e a gerência são fundamentais. No entanto, a supervisão individual tende a priorizar a resolutividade, "não deixar o problema aumentar"; a reunião da equipe privilegia o caso individual e a doença; a gerência da unidade costuma ter uma atuação muito pequena na conformação do trabalho da equipe e do agente. Quando se considera o plano objetivo do Programa, o agente se vê como educador para a saúde, organizador de acesso (cadastrador e orientador do uso de serviços) $e$ "olheiro" da equipe na captação de necessidade, identificação de prioridades e detecção de casos de risco para intervenção da equipe (Silva, 2001). Se a identidade do agente pende mais freqüentemente para o pólo técnico, aquele das ações da instituição e da assistência ao indivíduo, como evidenciado em vários estudos, a equipe, na sua função de gerência da proposta, envolvendo a supervisão do médico e do enfermeiro, e mesmo o trabalho formal do gerente da unidade, parece pouco ver as atribuições desses dois pólos ou orientar mais um ou outro. A variedade de concepções e entendimentos sobre o agente comunitário de saúde e sobre a sua função, de que são portadores os demais membros da equipe do PSF, os diretores de unidades, enfim, os demais sujeitos dos Programas, evidencia a dimensão das expectativas a que ele teria que atender no seu dia-a-dia e, por conseqüência, algumas condições concretas de conformação de uma identidade.

\section{Os desafios para o saber fazer e o saber ser}

As considerações anteriores apontam alguns desafios para a atuação do ACS, sistematizados aqui em seis pontos, sem preocupação com a ordem de relevância: o contexto, a finalidade, a tecnologia, o trabalho em equipe, a identidade e a formação.

Em primeiro lugar, há que se considerar a enorme variedade de contexto 
em que se implanta o Programa, exigindo flexibilização em sua operação e, por conseqüência, nos processos e metodologias de preparação de pessoal. Como segundo ponto, há que se considerar a amplitude das finalidades do Programa, agregando aspectos não apenas baseados em formas de atuação distintas, mas muitas vezes antagônicas. É exemplo disso a compreensão de uma parte significativa dos problemas, a partir de sua vertente mais individual e biológica, ou mais coletiva e social; ou, em outros termos, uma vertente mais de vigilância a situações de risco e assistência a doenças mais prevalentes ou aquela da promoção da saúde e da qualidade de vida. Além da questão da apreensão, há uma disputa pelo tempo de trabalho dedicado por cada profissional às suas diferentes funções. Em geral, quando há uma competição entre diferentes ações, aquelas cuja demanda é maior e têm uma rotina estabelecida, tendem a representar a parte maior do investimento do trabalhador. Considerando as diferentes funções do agente, pode-se identificar um relativo destaque daquelas relacionadas com a vigilância à saúde na sua vertente mais individualizada e clínica. Se, de um lado, esse é um aspecto importante do trabalho, pela sua potencialidade de identificar situações de maior vulnerabilidade individual (Mann et al., 1993), sabe-se que as ações baseadas em estratégias de grupo de risco têm um impacto reduzido na qualidade de saúde da população (Rose, 1988). Por outro lado, se a promoção da saúde representa uma modalidade de atuação promissora para melhorar a qualidade de vida (Gentile, 1999; Buss, 2000), ela carrega em si uma série de desafios. Sendo um campo recente de atuação, o conhecimento e a prática não estão sedimentados; de outro, para se produzir resultados, há necessidade de articular ações para além do campo da assistência à saúde, tecendo-se a rede das ações intersetoriais (Teixeira \& Paim, 2000). Portanto, para uma efetiva mudança no quadro de saúde da população, um terceiro aspecto é desenvolver uma tecnologia de trabalho adequada às necessidades (Schraiber et al., 1996) não só na área da atenção primária, mas no plano das políticas públicas. Neste ponto, as questões são das mais problemáticas, desde a concepção de família $e$ a abordagem adequada a essa, até as relações sociais em áreas urbanas $e$ rurais, entre as quais está inserida a unidade básica e a equipe de saúde da família. Definir as competências e desenvolver os instrumentos de trabalho correspondentes exige prioridade. Entre as atividades dos agentes, a do cadastramento é considerada como relativamente mais bem estabelecida do que o conteúdo das visitas subseqüentes (Silva, 2001). No entanto, mesmo para o cadastramento, além da capacitação do agente para levantar os dados preconizados pelo Programa hoje em nível nacional, há que se contar com uma observação apropriada das relações interpessoais e uma contribuição para discussão com a equipe das necessidades de saúde das famílias. Para as visitas posteriores, se o objetivo é trabalhar com as famílias $e$ a comunidade, identificam-se hoje determinadas estratégias como a valorização do diálogo - a conversa como instrumento de trabalho - e do apoio social, nas suas vertentes informação, empoderamento e instrumental (Robertson \& Minkler, 1994). Para as relações sociais, os princípios da solidariedade e da cidadania são marcos para o trabalho. No entanto, se é 
possível contar com objetivos gerais estabelecidos, para implantar uma oferta organizada de ações (Paim, 1995), há que se fazer desdobramentos dos princípios para a atividade cotidiana, conformando o saber, os instrumentos $e$ as formas de atuar (Mendes-Gonçalves, 1994), e preparando os sujeitos da prática. Daí emerge o quarto ponto, no caso a constituição de uma equipe integradora (Peduzzi, 1998), que elabore um projeto de trabalho voltado para a promoção da saúde e para a articulação da unidade básica com outros equipamentos e movimentos sociais. Quando o PSF é implantado em uma região com falta de estrutura de retaguarda para atenção médica especializada, grande parte dos esforços para integração se dá em torno do acesso à assistência individual. Um quinto aspecto, muito importante para o agente, é o da sua identidade. Silva (2001) constatou que, em situações concretas de ação e interação, o agente comunitário compõe dimensões técnicas e políticas do trabalho, pendendo mais para um dos pólos, institucional ou comunitário. Se uma parte significativa dos agentes considera o seu trabalho gratificante, quer pela possibilidade de ser útil, quer pelo apoio a uma população carente, sua atuação implica envolvimento pessoal e desgaste emocional. Espera-se do agente uma atuação no contexto social, tanto na participação popular, como na abordagem de problemas que escapam à dimensão estrita da saúde biológica, como a violência. O agente muitas vezes refere ansiedade tanto na sua relação com a comunidade como com a equipe, especialmente quando se sente pressionado entre ambos. Cabe lembrar que a não definição de uma tecnologia adequada às necessidades e finalidades do trabalho contribui para esse permanente foco de tensão entre as dimensões da prática, e para a alternativa de se hipertrofiar aquela mais técnica, de localização institucional.

Como um sexto ponto, destaca-se a formação dos profissionais para a saúde da família, quando o maior investimento tem sido feito na preparação dos profissionais universitários, médicos e enfermeiros. Se, habitualmente, a qualificação do auxiliar de enfermagem privilegia as funções mais comuns em hospital, como os procedimentos de coleta de exame, aplicação de injetáveis, curativos etc, na atenção primária existe um campo extenso de atividades educativas e de aconselhamento que demandam uma habilitação adequada. O agente comunitário, por sua vez, além do treinamento introdutório, um pouco mais abrangente, participa (quando elas acontecem) de discussões temáticas conduzidas por médicos e enfermeiros no nível local ou regional. Nos espaços de educação continuada, encontram-se com freqüência os conteúdos tradicionais de conhecimento $e$ prática na área da saúde, havendo dificuldade de se dar conta da totalidade das finalidades colocadas para o PSF.

Uma última contribuição ao debate: quais seriam as estratégias para o desenvolvimento do trabalho do agente comunitário? Elencam-se algumas que aparecem, hoje, como mais produtivas: 1 o desenvolvimento de planos integrados para a área social comprometidos com a eqüidade; 20 envolvimento maior dos agentes e de parte da carga horária da equipe com atividades coletivas e comunitárias; 3 um investimento maior em atividades de supervisão dos trabalhos, pois se a formação 
básica ou o treinamento específico é necessário, a manutenção da qualidade do trabalho se faz mediante atividades de supervisão e de reflexão; 4 considera-se que a supervisão dos agentes comunitários deveria cobrir os diferentes ângulos do seu trabalho: as visitas domiciliares, com especial atenção para os casos/ casas que constituem situações de maior vulnerabilidade; as atividades comunitárias, que podem ter sua origem em programas da área social ou serem atividades reivindicativas de direitos de cidadania; a situação de trabalhador do agente, identificando sofrimento e apoiando formas de lidar com conflitos; 5 finalmente, para a construção de novas práticas e do conhecimento correspondente, há que se investir sempre em pesquisa, quer de natureza mais operacional, na avaliação de processos e resultados, quer de teorias acerca do trabalho e da sua organização.

Nessa perspectiva, há necessidade de desenvolvimento e incorporação de tecnologias que apóiem a identidade do agente comunitário, integrando as diferentes dimensões de sua atuação - as previstas $e$ as necessárias - $e$ de preparação de todos os demais sujeitos do Programa, e não apenas o agente comunitário de saúde.

Para concluir, quando se colocam em questão o trabalho e o saber do agente comunitário de saúde, parece-nos ainda muito atual, para 2002, lembrar e ressaltar a propriedade do modelo sugerido para a capacitação dos agentes de saúde do Projeto do Vale do Ribeira, em São Paulo, na primeira metade da década de oitenta (Silva, 1984) que, segundo os coordenadores, visava atingir duas preocupações centrais:

a primeira, fortalecer o compromisso e a solidariedade do agente de saúde com a comunidade e a segunda, prover condições para a apropriação, pelo agente de saúde, do instrumental adequado e necessário para lidar com os problemas de saúde do grupo.

(Silva, 1984, p.33)

Referências

ABBOTT P., WALLACE C. The sociology of the caring professions. London: The Falmer Press, 1990.

BRASIL. Ministério da Saúde. Fundação Nacional de Saúde. Programa Nacional de Agentes Comunitários de Saúde. Manual do Agente Comunitário de Saúde. Brasília, 1991.

BRASIL. Ministério da Saúde. Programa Comunidade Solidária. Programa de Agentes Comunitários de Saúde. Programa de Agentes Comunitários de Saúde. Brasília, 1997.

BRASIL, Ministério da Saúde. Secretaria de Assistência à Saúde. Manual para a Organização da Atenção Básica. Brasília, 1999a.

BRASIL, Ministério da Saúde. Coordenação Geral de Desenvolvimento de Recursos Humanos para o SUS/ SPS/MS. Coordenação de Atenção Básica/SAS/MS. Diretrizes para elaboração de programas de qualificação e requalificação dos Agentes Comunitários de Saúde. Brasília, 1999b.

BUSS, P. M. Promoção da saúde e qualidade de vida. Ciênc. Saúde Coletiva, v. 5, n.1, p. 163-77, 2000.

DONNANGELO, M.C.F., PEREIRA, L. Saúde e Sociedade. São Paulo: Duas Cidades, 1976.

GENTILE, M. Promoção da Saúde. Rev. Minist. Saúde, Secretaria de Políticas de Saúde, ano 1, n.1, p. 911, 1999. 
JATENE, AD., NOVAES, H.M.D., MALIK, A., GOLDBAUM, M., MARSIGLIA, R.G., SILVA, J.A, SEIXAS, P. Primeiro Relatório Técnico-Científico: novos modelos de Assistência à Saúde Avaliação do Programa de Saúde da Família no Município de São Paulo. São Paulo: FAPESP, 2000.

MANN, J., TARANTOLA, D.J.M., NETTER, T.W. Como avaliar a vulnerabilidade à infecção pelo HIV e AIDS. In: A AIDS no mundo. Rio de Janeiro: Relume Dumará: ABIA: IMS, UERJ, 1993, p.275-300.

MENDES-GONÇALVES, R.B. Tecnologia e organização social das práticas de Saúde: características tecnológicas do processo de trabalho na rede estadual de Centros de Saúde de São Paulo. São Paulo: Editora HUCITEC/ABRASCO, 1994.

NOGUEIRA, R., RAMOS, Z.V.O. A vinculação institucional de um trabalhador sui generis - 0 agente comunitário de saúde. [Texto para discussão 735]. 2000. 33p. Disponível em <URL: http:// www.ipea.gov.br >. Acesso em: jun 2000.

PAIM, J.S. A reorganização das práticas em distritos sanitários. In: Mendes (Org.) Distrito sanitário: o processo social de mudança das práticas sanitárias do Sistema Único de Saúde. São Paulo- Rio de Janeiro: HUCITEC-ABRASCO, 1995. p.187-220.

PEDUZZI, M. Equipe multiprofissional de saúde: a interface entre trabalho e interação. Campinas, 1998. Tese (Doutorado) Faculdade de Ciências Médicas, Universidade Estadual de Campinas.

ROBERTSON, A., MINKLER, M. New Health promotion movement: a critical examination. Health Education Quarterly, v.21, n.3, p. 295-312, 1994.

ROSE, G. Individuos enfermos y poblaciones enfermas.In: BUCK, C. (Org.) El desafio de la epidemiologia: problemas y lecturas seleccionadas. Washington: OPS, 1988. p.900-9. (Publicacion cientifica 505)

SCHRAIBER, L., NEMES, M.I.B., MENDES-GONÇALVES R.B. (orgs). Necessidades de saúde e atenção primária. In: Saúde do Adulto: programas e ações na Unidade Básica. São Paulo: HUCITEC, 1996. p.29-47.

SILVA, J.A. Assistência Primária de Saúde: o agente de Saúde do Vale do Ribeira. São Paulo, 1984. Dissertação (Mestrado) Faculdade de Saúde Pública, Universidade de São Paulo.

SILVA, J.A O agente comunitário de saúde do Projeto QUALIS: agente institucional ou agente de comunidade? São Paulo, 2001. Tese (Doutorado) Faculdade de Saúde Pública, Universidade de São Paulo.

TEIXEIRA, C.F., PAIM, J.S. Planejamento e programação de ações intersetoriais para a promoção da saúde e da qualidade de vida. Rev. Adm. Pública, v.34, n.6, p.63-80, 2000. 